\title{
Ventriculo-atrial time interval measured on $M$ mode echocardiography: a determining element in diagnosis, treatment, and prognosis of fetal supraventricular tachycardia
}

\author{
E Jaeggi, J C Fouron, A Fournier, N van Doesburg, S P Drblik, F Proulx
}

\begin{abstract}
Objective-To determine whether $M$ mode echocardiography can differentiate fetal supraventricular tachycardia according to the ventriculo-atrial (VA) time interval, and if the resulting division into short and long VA intervals holds any relation with clinical presentation, management, and fetal outcome.

Design-Retrospective case series.
\end{abstract}

Subjects-23 fetuses with supraventricular tachycardia.

Main outcome measures-A systematic review of the $M$ mode echocardiograms (for VA and atrioventricular (AV) interval measurements), clinical profile, and final outcome.

Results-19 fetuses (82.6\%) had supraventricular tachycardia of the short VA type (mean (SD) VA/AV ratio $0.34(0.16)$; heart rate 231 (29) beats/min). Tachycardia was sustained in six and intermittent in 13. Hydrops was present in three $(15.7 \%)$. Digoxin, the first drug given in 14 , failed to control tachycardia in five. Three of these then received sotalol and converted to sinus rhythm. All fetuses of this group survived. Postnatally, supraventricular tachycardia recurred in three, two having Wolff-Parkinson-White syndrome. Four fetuses (17.4\%) had long VA tachycardia (VA/AV ratio $3.89(0.82)$; heart rate 226 (10) beats/min). Initial treatment with digoxin was ineffective in all, but sotalol was effective in two. Heart failure caused fetal death in one and premature delivery in one. All three surviving fetuses had recurrences of supraventricular tachycardia after birth: two had the permanent form of junctional reciprocating tachycardia and one had atrial ectopic tachycardia.

Conclusions-Careful measurement of ventriculo-atrial intervals on fetal $M$ mode echocardiography can be used to distinguish short from long VA supraventricular tachycardia and may be helpful in optimising management. Digoxin, when indicated, may remain the drug of choice in the short VA type but appears ineffective in the long VA type.

(Heart 1998;79:582-587)

Keywords: fetus; supraventricular tachycardia; ventriculo-atrial interval; digoxin
Supraventricular tachycardia is the most commonly reported type of fetal tachycardia ${ }^{12}$ and may be associated with cardiac decompensation, manifested by ascites, pleural effusion, pericardial effusion, anasarca, and death. ${ }^{3-5}$ It usually requires aggressive treatment to restore sinus rhythm, thus eliminating the risk of cardiac failure. The most common mechanism that underlies supraventricular tachycardia in neonates and older children (excluding sinus tachycardia) is reentry, accounting for about $90 \%$ of all narrow QRS tachycardia. ${ }^{67}$ In infants, reentry circuits most often occur through accessory atrioventricular (AV) connections, while reentry within the AV node is less often observed. ${ }^{8-10}$ In typical reentry supraventricular tachycardia, antegrade conduction through the AV node is slow, while retrograde pathways show fast conducting properties. On the ECG, retrograde atrial activation occurs shortly after the QRS complex and thus yields a short retrograde pathway tachycardia. ${ }^{7}$ In other and less common forms of narrow QRS tachycardia, the retrograde limb shows slow conduction properties and results in a long retrograde pathway tachycardia; this is the case in the permanent form of junctional reciprocating tachycardia, which is also the most commonly observed type of long retrograde pathway tachycardia. ${ }^{11}$ In atrial ectopic tachycardia there is no reentry circuit and the $\mathrm{P}$ wave always precedes the QRS complex, resulting in a short PR interval and a long RP interval. This type of arrhythmia is very uncommon in infancy. ${ }^{12}$ The permanent form of junctional reciprocating tachycardia and atrial ectopic tachycardia are usually incessant and tend to be unresponsive to most antiarrhythmic agents. ${ }^{13}$ Thus the further differentiation of fetal supraventricular tachycardias into those with long and short retrograde pathways could be useful for optimal treatment. In this study, we investigated whether careful measurements of atrioventricular and ventriculo-atrial (VA) intervals on $M$ mode echocardiograms recorded during fetal tachycardia could help establish the type of VA interval associated with the tachycardia, and whether this distinction is relevant to the clinical presentation, management, and outcome of the affected fetuses.

\section{Methods}

DEFINITIONS

Intrauterine supraventricular tachycardia was defined by a 1:1 AV conduction relation with a heart rate of more than 180 beats $/ \mathrm{min}$ and little or no beat to beat variation. 

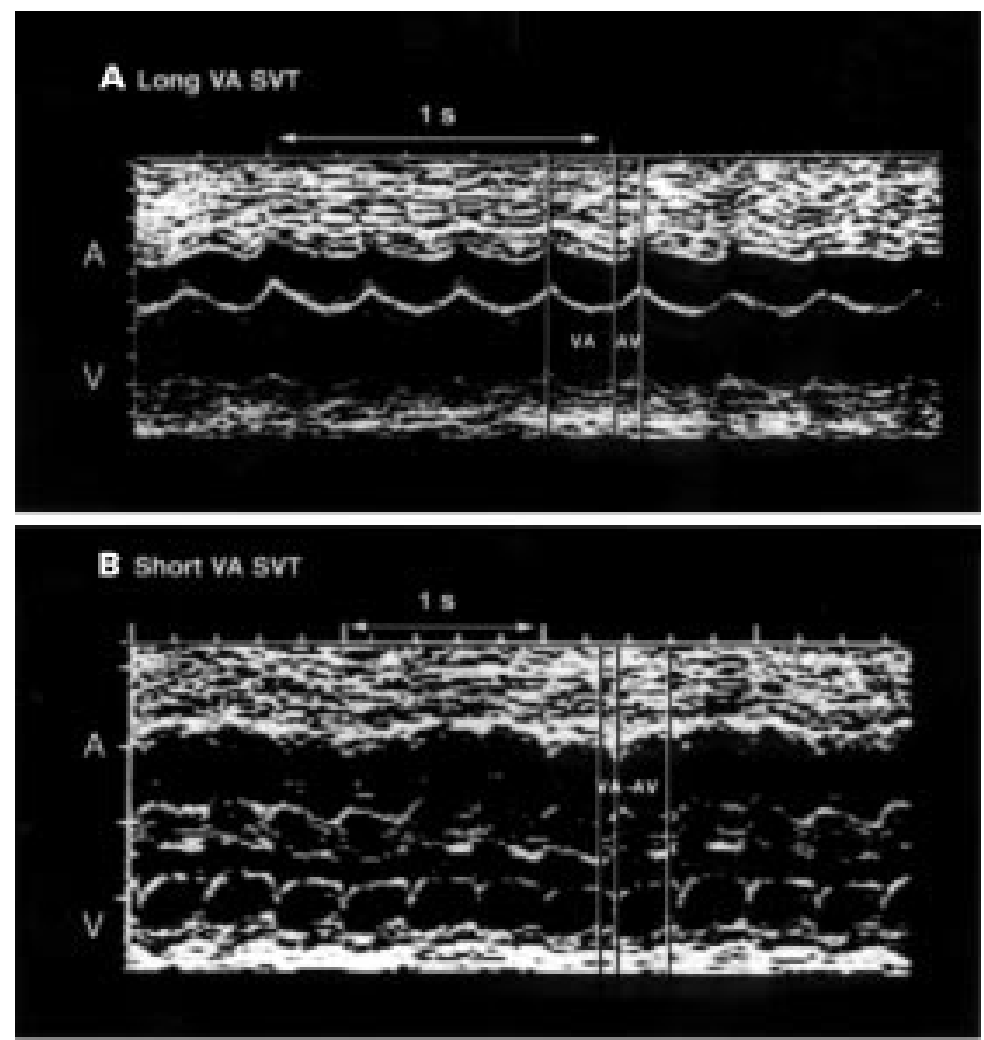

Figure 1 Measurement of ventriculo-atrial time interval on $M$ mode echocardiography during fetal supraventricular tachycardia. Examples of a long (panel A) and short (panel $B$ ) ventriculo-atrial time intervals are shown. A, atrium; $V$, ventricle.
Supraventricular tachycardia was considered sustained when observed permanently or when present for more than $50 \%$ of the time required for the echocardiographic examination (approximately 45 minutes); it was classified as intermittent if the tachycardia lasted less than $50 \%$ of the time. The diagnosis of congestive heart failure was based on the presence of cardiomegaly and fluid accumulation in at least one of the pleural, pericardial, or abdominal cavities. The diagnosis of tachycardia related cardiomyopathy was made when marked thickening and hyperechogenicity of the myocardium were associated with poor contractility. Diagnosis of hydrops fetalis required the presence of fluid collections in at least two of the abdominal, pleural, or pericardial spaces and/or anasarca. ${ }^{5}$ In this clinical situation, it was considered to represent an advanced state of cardiac failure. After birth, the diagnosis of the permanent form of junctional reciprocating tachycardia and atrial ectopic tachycardia was made according to the electrocardiographic and clinical criteria reported previously. ${ }^{1214-16}$

CLINICAL MATERIAL

All cases of fetal supraventricular tachycardia seen between 1988 and 1995 at our fetal cardiology unit were systematically reviewed. Atrial flutter and multifocal tachycardia were excluded from the study. Fetuses who where already on antiarrhythmic treatment before the first echocardiographic study were also excluded. Supraventricular tachycardia was documented in 27 subjects on at least one

Table 1 Summary of patient characteristics with short and long VA interval

\begin{tabular}{|c|c|c|c|c|c|c|c|c|c|c|}
\hline Case & $V A / A V$ ratio & $\begin{array}{l}\text { GA at } \\
\text { detection }\end{array}$ & $\begin{array}{l}\text { Max HR } \\
\text { (beats/min) }\end{array}$ & $\begin{array}{l}\text { Pattern of } \\
\text { SVT }\end{array}$ & $\begin{array}{l}\text { Sign of } \\
\text { CHF }\end{array}$ & Treatment in utero & GA at birth & Treatment postnatal & $\begin{array}{l}\text { SVT } \\
\text { postnatal }\end{array}$ & $E C G$ \\
\hline \multicolumn{11}{|c|}{ Short VA SVT } \\
\hline 1 & 0.55 & 26 & 240 & Sustained & $\mathrm{H}$ & $\mathrm{D}(-), \mathrm{S}(+)$ & 40 & $\mathrm{~S}(+)$ & Yes/reentry & WPW \\
\hline 2 & 0.52 & 35 & 200 & Sustained & No & $\begin{array}{l}\mathrm{D}(-) ; \mathrm{V}(-) \text {, } \\
\text { delivery }\end{array}$ & 37 & $\operatorname{Propr}(+)$ & No & WPW \\
\hline 3 & 0.34 & 31 & 288 & Intermittent & No & $\mathrm{D}(+)$ & 40 & No & No & Normal \\
\hline 4 & 0.22 & 30 & 214 & Intermittent & No & $\mathrm{D}(-) ; \mathrm{S}(+)$ & 40 & No & No & Normal \\
\hline 5 & 0.33 & 31 & 187 & Intermittent & No & $\mathrm{D}(+)$ & 41 & $\mathrm{D}(+)$ & No & Normal \\
\hline 6 & 0.3 & 25 & 192 & Sustained & No & $\mathrm{D}(+)$ & 40 & $\mathrm{D}(-), \mathrm{S}(+)$ & Yes/reentry & Normal \\
\hline 7 & 0.44 & 34 & 200 & Intermittent & No & No & 40 & No & No & Normal \\
\hline 8 & 0.22 & 31 & 219 & Intermittent & $\mathrm{PE}$ & $\mathrm{D}(+)$ & 40 & $\mathrm{D}(+)$ & No & Normal \\
\hline 9 & 0.49 & 20 & 218 & Intermittent & No & $\mathrm{D}(-)$ & 40 & $\mathrm{D}(+)$ & No & Normal \\
\hline $10^{\star}$ & 0.32 & 32 & 229 & Intermittent & No & No & 33 & No & No & Normal \\
\hline 11 & 0.15 & 38 & 222 & Intermittent & No & No & 40 & No & No & Normal \\
\hline 12 & 0.63 & 38 & 230 & Sustained & No & No & 38 & $\mathrm{D}(+)$ & No & WPW \\
\hline $13^{\star}$ & 0.44 & 25 & 230 & Intermittent & No & $\mathrm{D}(+)$ & 38 & No & No & Normal \\
\hline 14 & 0.07 & 36 & 272 & Sustained & No & $\mathrm{D}(+)$ & 39 & $\mathrm{D}(+)$ & No & Normal \\
\hline $15 \dagger$ & 0.35 & 30 & 214 & Intermittent & No & $\mathrm{D}(+)$ & 39 & No & No & RVH \\
\hline $16^{\circ}$ & 0.33 & 32 & 250 & Intermittent & No & $\mathrm{D}(+)$ & 38 & $\mathrm{D}(+)$ & No & Normal \\
\hline 17 & 0.55 & 28 & 260 & Intermittent & $\mathrm{H}, \mathrm{CMP}$ & $\mathrm{D}(+)$, delivery & 32 & $\mathrm{D}(+)$ & No & Normal \\
\hline 18 & 0.05 & 26 & 232 & Intermittent & No & No & 39 & No & No & Normal \\
\hline 19 & 0.16 & 32 & 285 & Sustained & $\mathrm{H}$ & $\begin{array}{l}\mathrm{D}(-), \mathrm{S}(+) \\
\text { delivery }\end{array}$ & 35 & $\mathrm{~S}(+)$ & Yes/reentry & WPW \\
\hline Mean (SD) & $0.34(0.16)$ & $30.5(4.7)$ & $230.6(29)$ & & $3 / 19$ & $(+)=17 / 19$ & $38.4(2.5)$ & & $3 / 14$ & \\
\hline \multicolumn{11}{|c|}{ Long VA SVT } \\
\hline 20 & 3.13 & 24 & 234 & Sustained & $\mathrm{H}, \mathrm{CMP}$ & $\mathrm{D}(-), \operatorname{Ad}(-), \mathrm{S}(+)$ & Death iu & & - & \\
\hline 21 & 3.33 & 27 & 235 & Sustained & No & $\mathrm{D}(-), \mathrm{S}(-)$, delivery & 36 & $\begin{array}{l}\mathrm{D}(-), \mathrm{S}(-) \\
\operatorname{Flec}(-), \operatorname{Pf}(+)\end{array}$ & Yes/PJRT & Normal \\
\hline 22 & 4.23 & 29 & 220 & Sustained & No & $\mathrm{D}(-), \mathrm{S}(+)$ & 40 & $\mathrm{D}(-), \mathrm{S}(+)$ & Yes/PJRT & Normal \\
\hline 23 & 4.89 & 34 & 216 & Sustained & $\mathrm{CMP}$ & $\mathrm{D}(-)$, delivery & 35 & $\begin{array}{l}\mathrm{D}(-), \operatorname{Ad}(-), \\
\mathrm{EC}(-), \operatorname{Am}(+), \operatorname{Pf}(+)\end{array}$ & Yes/AET & Normal \\
\hline Mean (SD) & $3.89(0.82)$ & $28.5(4.2)$ & $226(9.7)$ & & $2 / 4$ & $(+)=2 / 4$ & $37(2.6)$ & & $3 / 3$ & \\
\hline
\end{tabular}

Drug effects: (-), no rhythm control; (+), rhythm control.

*Twin pregnancy; ttetralogy of Fallot.

$\mathrm{CHF}$, congestive heart failure; CMP, cardiomyopathy; GA, gestational age in weeks; $\mathrm{H}$, hydrops; HR, maximum heart rate before treatment; iu, in utero; PE, pericardial effusion, RVH, right ventricular hypertrophy; SVT, supraventricular tachycardia; WPW, Wolff-Parkinson-White syndrome; PJRT, permanent form of junctional reciprocating tachycardia; AET, atrial ectopic tachycardia.

Ad, adenosine; Am, amiodarone; D, digoxin; EC, electroconversion; Flec, flecainide; Pf, propafenone; Propr, propranolol; S, sotalol; V, verapamil. 


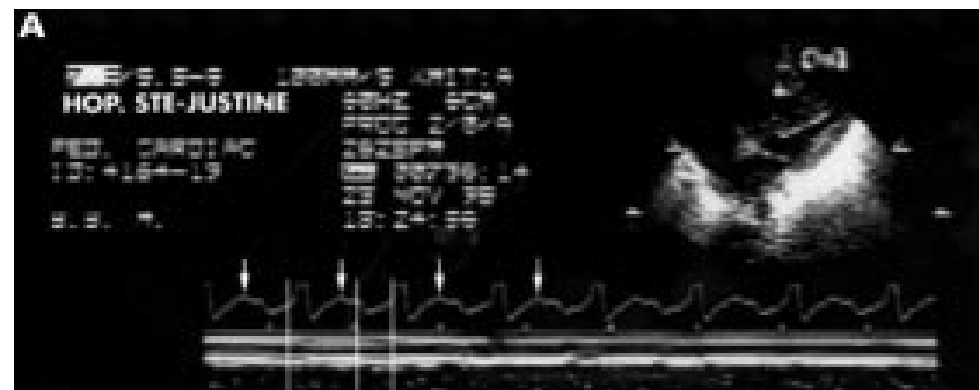

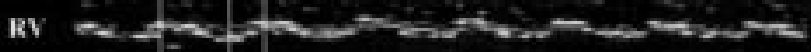

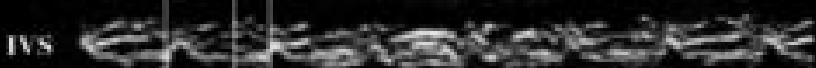

$\mathrm{MV}-2$ as va.

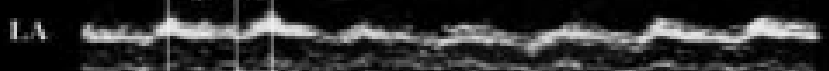

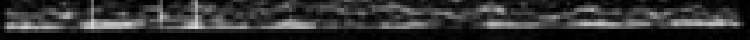
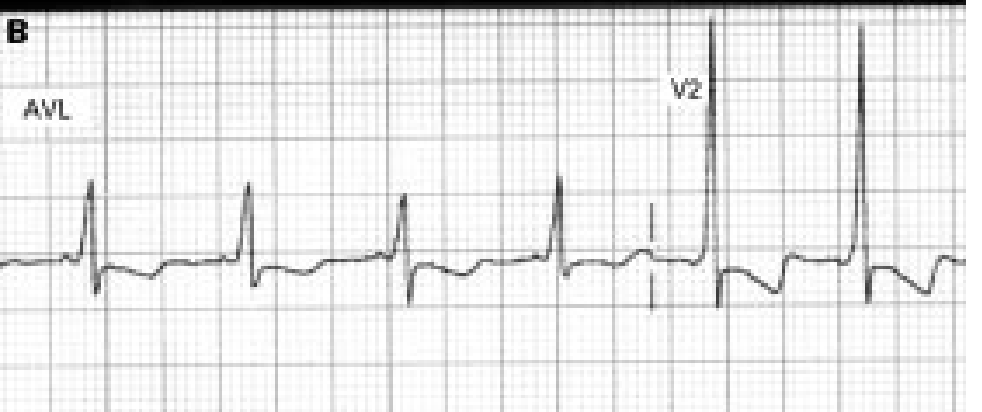

Figure 2 (A) Example of simultaneous $M$ mode echocardiogram and ECG recordings during a neonatal reentry supraventricular tachycardia. Arrows mark peaks of $P$ waves. Vertical lines mark mechanical ventricular and atrial events. The ECG shows a short RP supraventricular tachycardia of 260 beats/min with 1:1 AV conduction, an RP interval of $95 \mathrm{~ms}$, and a PR interval of $135 \mathrm{~ms}$, leading to an electrical $R P / P R$ ratio of 0.7 . The $M$ mode echocardiogram indicates a corresponding short VA tachycardia with a VA interval of $80 \mathrm{~ms}$, an AV interval of $150 \mathrm{~ms}$, and a mechanical VA/AV ratio of 0.53. (B) Electrocardiographic demonstration of pre-excitation (Wolff-Parkinson-White syndrome) after termination of the supraventricular tachycardia in the same neonate (case 19). IVS, interventricular septum; $L A$, left atrium; $M V$, mitral valve; $R V$, right ventricle.

echocardiogram. Four cases were excluded for one of the reasons mentioned.

\section{PROCEDURES}

Fetal echocardiographic imaging and $M$ mode examinations were performed with 3.5 and 5.0 $\mathrm{MHz}$ transducers using either an HP Sonos 100 (Hewlett-Packard, Palo Alto, California, USA) or an Acuson Computed Sonographic machine model 128XP/10c (Acuson, Mountain View, California, USA). The $M$ mode tracings of the 23 remaining fetuses recorded before any drug treatment were reviewed blindly by an experienced echocardiographer (EJ) not aware of the clinical history and the outcome of the patients.

Guided by bidimensional real time imaging, it was always possible to direct the $M$ mode ultrasound beam simultaneously through the atrial and ventricular walls. VA and AV intervals, analogous to the RP and PR intervals of the extrauterine electrocardiogram, were measured at peak atrial and ventricular systolic wall motions. A long VA tachycardia was defined by a VA/AV ratio above 1 . The cardiothoracic ratio was obtained on the four chamber view by dividing the cardiac by the thoracic circumferences. Cardiomegaly was defined as a cardiothoracic ratio greater than two standard deviations above the mean for gestational age. ${ }^{17}$

The clinical records of each of these fetuses was then reviewed for the following variables: gestational age at detection of supraventricular tachycardia, maximum heart rate before treatment, presence of congestive heart failure, type of initial treatment and response, the need for more than one drug, and recurrence of supraventricular tachycardia on follow up. Postnatal evaluation included gestational age at birth, presence of cardiac malformations, and an electrocardiographic study during sinus rhythm and, when present, during episodes of tachycardia.

According to our management protocol, antiarrhythmic treatment with maternal digoxin was indicated when congestive heart failure or sustained supraventricular tachycardia was present. Mothers received loading doses of $2 \mathrm{mg}$ digoxin over a two day period, followed by a maintenance dose of 0.5 to $0.75 \mathrm{mg}$ daily, adjusted to maintain maternal digoxin concentrations near $2 \mathrm{nmol} / \mathrm{l}$. If digoxin appeared to be ineffective after eight days of treatment, oral sotalol ( 80 to $160 \mathrm{mg}$ twice a day) was added in fetuses younger than 35 gestational weeks, or the pregnancy was electively terminated when the fetus was older.

\section{DATA ANALYSIS}

Patients were divided into two groups based on the presence of short or long VA intervals during fetal tachycardia. The Student $t$ test was used to compare means, while the Fisher exact test was used to analyse categorical variables. Statistical significance was set at $\mathrm{p}=0.05$.

\section{Results}

$M$ mode echocardiograms allowed the identification of 19 fetuses with a short VA interval, with a mean (SD) VA/AV ratio of $0.34(0.16)$, and four with a long VA interval, with a mean $\mathrm{VA} / \mathrm{AV}$ ratio of $3.89(0.82)(\mathrm{p}<0.003)$. The intra- and interobserver variabilities on 40 measurements were $13.1(14.1) \%$ and $16.6(13.9) \%$ for VA, and $7.5(5.3) \%$ and $10.2(6.6) \%$ for AV, respectively. Figure 1 shows an example of the $M$ mode echocardiograms recorded from each group. The clinical presentation, management, and outcome are presented in table 1 .

\section{CLINICAL PRESENTATION}

The two groups of fetuses did not differ significantly in mean gestational age at detection of supraventricular tachycardia (30.5 (4.7) $v 28.5$ (4.2) weeks for the short and long VA interval groups, respectively) and heart rate before medical treatment (230.6 (29) $v$ 226 (10) beats/min). The initial ultrasound examinations showed an intermittent tachycardia in 13 of the 19 fetuses (68\%) with a short VA interval. Supraventricular tachycardia with a short VA interval led to hydrops in three cases, with cardiomyopathy in one. One cardiac malformation was observed in the short VA group. In contrast, all four fetuses with long VA intervals showed the same pattern of sustained tachycardia. This difference was 

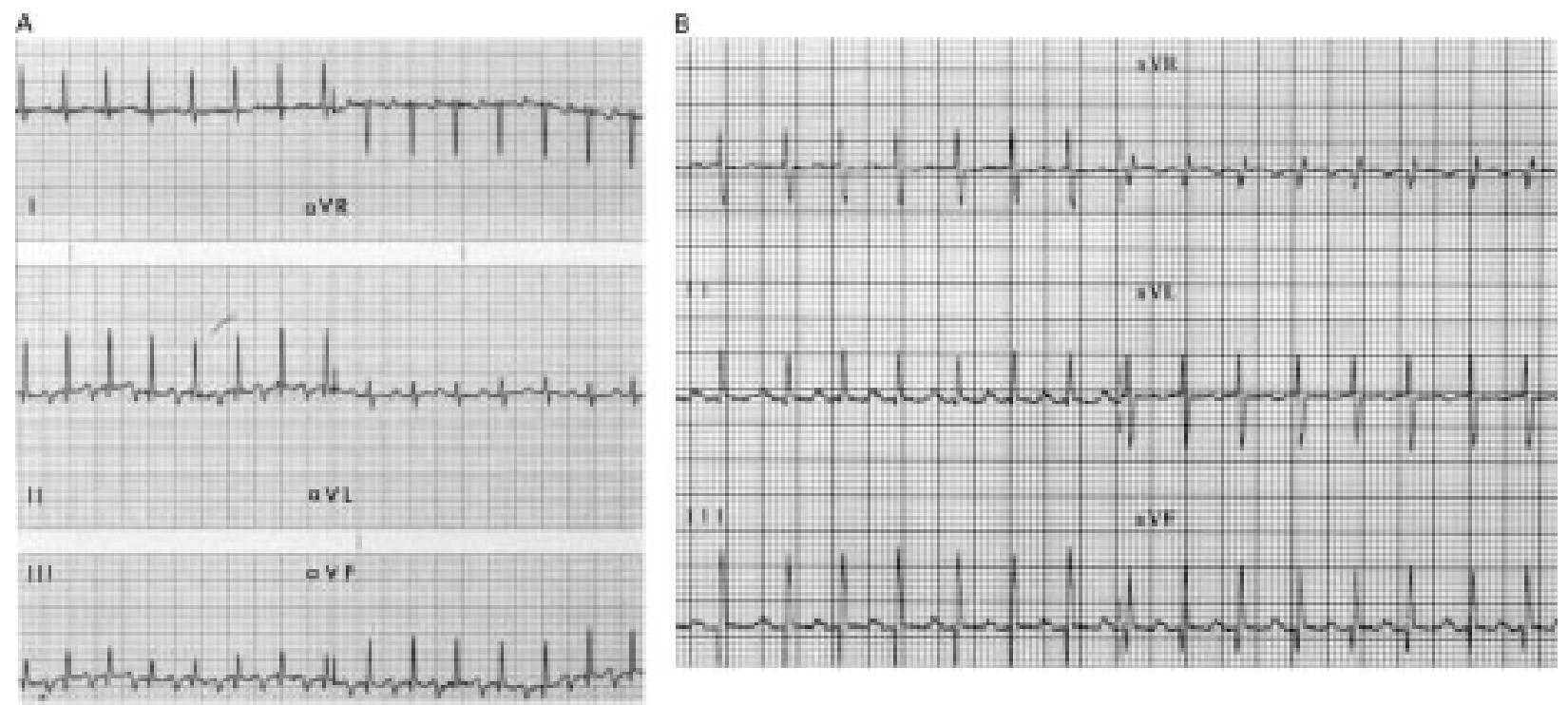

Figure 3 (A) Electrocardiogram of one of the neonates with permanent form of junctional reciprocating tachycardia: limb leads I, II, III, aVR, aVL, aVF in full standardisation; narrow QRS tachycardia at 180 beats/min with l:l AV relation; long RP, short PR; negative P waves in II, III, and aVF (B) Atrial ectopic tachycardia: limb leads I, II, III, aVR, aVL, aVF in full standardisation, narrow QRS tachycardia at 180 beats/min with l:l AV relation; long RP, short PR; abnormal P wave morphology and axis.

statistically significant $(\mathrm{p}<0.003)$. Two fetuses in this group showed evidence of tachycardia induced cardiomyopathy.

FETAL MANAGEMENT AND OUTCOME

Short VA group

Drug treatment was indicated in 14 of the 19 fetuses in the short VA group. Digoxin, always the first drug given, successfully controlled tachyarrhythmia in nine fetuses (64\%), while it failed to convert the tachycardia in five (cases $1,2,4,9$, and 19). In three instances (cases 1 , 4 , and 19) conversion to sinus rhythm was achieved before delivery by adding sotalol. Case 9 presented with intermittent supraventricular tachycardia, which persisted despite digoxin treatment but never showed signs of cardiac failure.

In four cases from this group, pregnancy had to be terminated prematurely, twice (cases 10 and 17) for maternal reasons. Patient 19 had to be delivered despite conversion to sinus rhythm with sotalol because of evidence of restrictive foramen ovale causing hypodevelopment of the left ventricle. Fetus 2 was at 35 weeks gestation at the time of diagnosis and was the only case delivered prematurely for lack of response to antiarrhythmic treatment.

In this group, drug treatment was considered unjustified in five fetuses, either because of the intermittent nature of the tachycardia (cases 7 , 10,11 , and 18) or because the diagnosis was made close to term and the fetus delivered before treatment (case 12). No patient in this group presented signs of congestive heart failure at birth.

After birth, eight infants with short VA remained on digoxin treatment for up to 12 months, while propranolol was given in one and sotalol in two. Recurrence of supraventricular tachycardia occurred in three infants (cases 1, 6, and 19), two of whom had Wolff-Parkinson-White syndrome. Surface ECGs showed Wolff-Parkinson-White in four of the 19 patients with short VA supraventricular tachycardia $(21 \%)$. All patients survived.

The mean follow up for this group was 3.6 (2.4) years. Figure $2 \mathrm{~A}$ shows an example of simultaneous $M$ mode and ECG recordings during reentry tachycardia in a neonate with Wolff-Parkinson-White syndrome. This figure shows the similarity between time interval measurements on ECG and M mode echocardiographic recordings; after conversion to sinus rhythm this patient showed a pre-excitation wave (Wolff-Parkinson-White syndrome) (fig 2B).

\section{Long VA group}

All four fetuses with long VA tachycardia required drug treatment. Digoxin, which was the drug of choice for all fetal supraventricular tachycardias before this study, failed to convert the tachycardia in all four of these cases. Compared with the short VA group, this lack of response was significant $(\mathrm{p}<0.04)$. One fetus (case 23) was electively delivered by caesarean section without further medication because of signs of progressive cardiomyopathy. Sotalol was added in the remaining three cases and two of them (cases 20 and 22) converted to sinus rhythm. One of the sotalol responders (case 20) died in utero at 26 weeks gestation because of persistent severe secondary cardiomyopathy with generalised hydrops. This fetus had failed to respond to direct fetal administration of adenosine two weeks earlier. Necropsy examination revealed no congenital malformation. The difference in the incidence of tachycardia related cardiomyopathy in the long VA group compared with the short VA group $(2 / 4 v 1 / 19)$ did not reach statistical significance $(p<0.06)$.

All three surviving neonates from this group had recurrent episodes of supraventricular tachycardia $(\mathrm{p}<0.01)$, requiring the administration of various antiarrhythmic drugs (table 1). The permanent form of junctional reciprocating tachycardia was diagnosed in two (cases 
21 and 22) (fig 3A), and an atrial ectopic tachycardia in the other patient (case 23) (fig 3B). In all cases, digoxin was ineffective in preventing recurrence of tachycardia, while propafenone (two cases) or sotalol (one case) succeeded. After a mean follow up of 2.5 (1.9) years, only one child with the permanent form of junctional reciprocating tachycardia (case 22) could be weaned from treatment (at 1.5 years).

\section{Discussion}

Up to now, fetal $M$ mode echocardiography has been used to analyse the relative chronology of atrial and ventricular mechanical systoles, allowing rapid determination of atrioventricular conduction patterns. ${ }^{18-22}$ The reliable identification of fetal arrhythmias such as atrial or ventricular ectopia, atrial flutter, supraventricular tachycardia, and partial or complete atrioventricular block has thus been possible. In extrauterine life, surface ECG, transoesophageal electrocardiograms and, more invasively, electrophysiological studies, allow the division of narrow QRS supraventricular tachycardias into two groups, based on the duration of the RP interval. ${ }^{7}$ Our present study shows that the same analysis can be performed in utero using $M$ mode echocardiography. As can be seen from the results, the values of the VA/AV ratio in the two groups of fetuses were quite disparate, averaging $0.34(0.16)$ for the short VA group and $3.84(0.82)$ for the long VA group. This large difference between the two ratios decreases the risk of erroneous classification of the type of tachycardia, allowing for the fact that the peak of ventricular contraction can occasionally be difficult to identify with precision on $M$ mode.

A long VA supraventricular tachycardia was found in four of our patients $(17 \%)$, and was associated with an increased risk of morbidity and mortality. Of these fetuses, two developed arrhythmia related cardiomyopathy, one died in utero at 26 weeks gestation, and the other had to be delivered prematurely. None of the long VA patients responded to maternal digoxin administration, though maternal sotalol proved effective in controlling the supraventricular tachycardia in utero in two of the three cases where it was used. In the same group, adenosine was unsuccessful when given directly into the umbilical vein of one fetus (case 20 ), and intravenously to a neonate (case 23). After birth, all surviving long VA infants had recurrences of supraventricular tachycardia in the first months of life despite prophylaxis with digoxin.

In contrast, prenatal conversion to sinus rhythm was observed in 17 of the 19 cases with short VA interval, either spontaneously (five cases) or after administration of digoxin alone (nine cases), or in combination with sotalol (three cases). The fact, however, that five fetuses in this group did not respond to digoxin suggests that in some cases other elements besides the VA/AV measurements may have to be taken into account if treatment fails. Only one tachycardia related cardiomyopathy was observed in this group (case 17). The postnatal recurrence risk of supraventricular tachycardia was substantially less in the short VA group than in the long VA group $(15.7 \% v 100 \%$; $\mathrm{p}<0.01$ ).

Since the completion of this study our management protocol for fetal supraventricular tachycardia has been redesigned. Once the diagnosis of supraventricular tachycardia has been established, efforts are now made to measure the VA and AV intervals on $\mathrm{M}$ mode echocardiograms before giving any drugs. In the presence of a short VA interval, a reentry mechanism with slow anterograde and fast retrograde conduction is the most likely diagnosis and the conventional protocol is applied, with digoxin as the drug of first choice. In cases where conversion to sinus rhythm is not achieved with digoxin and adenosine,${ }^{23-27}$ there is no consensus in medical reports on alternative drugs. In the past, we have mainly used sotalol, a class III antiarrhythmic agent with some non-selective $\beta$ adrenergic blocking activity. The favourable response previously reported on the immature myocardium ${ }^{28}$ and the positive results that we and others ${ }^{29-31}$ have obtained, encourage us to continue with this drug. Potential proarrhythmic effects of sotalol reported in adults and also in paediatric patients $^{32}$ remain a source of concern, and this excludes its use as the drug of first choice in uncomplicated short VA supraventricular tachycardia.

There have been no controlled trials to document the superiority of a specific antiarrhythmic drug for long VA supraventricular tachycardias, which are usually sustained and unresponsive to most antiarrhythmic drugs. Class III agents are potential drugs of first choice. Because of the known side effects of amiodarone on thyroid function and its long half life, sotalol could be considered as a suitable alternative-alone if cardiac failure is absent, or in combination with digoxin if there is circulatory decompensation. Other types of antiarrhythmic drug, such as class IC antiarrhythmic agents, have been used with success in a limited number of cases of unresponsive fetal supraventricular tachycardia. ${ }^{33} 34$

Because of the relative rarity of long VA supraventricular tachycardia in the paediatric age group, it is not surprising that only four fetuses could be identified with this type of arrhythmia. This small number of cases does not allow us to draw definitive conclusions. Further studies are obviously needed to reach a clear consensus on the best way to approach the long VA fetal supraventricular tachycardia. Only a multicentre investigational protocol will help achieve this goal in the shortest period of time.

1 Kleinman CS, Copel JA, Weinstein EM, et al. In utero diagnosis and treatment of fetal supraventricular tachycardia. Semin Perinatol 1985;9:113-29.

2 Van Engelen AD, Weijtens O, Brenner JI, et al. Management, outcome and follow-up of fetal tachycardia. $7 \mathrm{Am}$ Coll Cardiol 1994;24:1371-5.

3 Bergmans MGM, Jonker GJ, Kock HC. Fetal supraventricular tachycardia: review of the literature. Obstet Gynecol Surv 1985;40:61-8.

4 Owen J, Colvin EV, Davis RO. Fetal death after successful conversion of fetal supraventricular tachycardia with digoxin and verapamil. Am f Obstet Gynecol 1988;158: 1169-70. 
5 Skoll MA, Sharland GK, Allan LD. Is the ultrasound definition of fluid collections in non-immune hydrops fetalis helpful in defining the underlying cause or predicting

6 Kleinman CS, Copel JA. Electrophysiological principles and fetal antiarrhythmic therapy. Ultrasound Obstet Gynecol 1991;1:286-97.

7 Josephson ME. Supraventricular tachycardias. In: Clinical cardiac electrophysiology: techniques and interpretation, 2 nd ed. Philadelphia: Lea and Febinger, 1993:181-274.

8 Ko JK, Deal BJ, Strasburger JF, et al. Supraventricular tachycardia mechanisms and their age distribution in pediatric patients. Am $\mathcal{F}$ Cardiol 1992;69:1028-32.

9 Benson DW, Dunnigan A, Benditt DG, et al. Transoesophageal study of infant supraventricular tachycardia: electrophysiologic characteristics. Am $\mathcal{F}$ Cardiol 1983;52:1002-6.

10 Weidling SN, Saul JP, Walsh EP. Efficacy and risks of medical therapy for supraventricular tachycardia in neonates cal therapy for supraventricular tachycar
and infants. Am Heart $\mathcal{f} 1996 ; 131: 66-72$.

11 Ludomirsky A, Garson A. Supraventricular tachycardia. In: Gillette PC, Garson A, eds. Pediatric arrhythmias: electrophysiology and pacing. Philadelphia: WB Saunders, 1990: physiology

12 Mehta AV, Sanchez GR, Sacks EJ, et al. Ectopic automatic tachycardia in children: clinical characteristics, management and follow-up. 7 Am Coll Cardiol 1988;11:379-85.

13 Sung RJ. Sustained supraventricular tachycardia. Pace 1983; 6:1306-26.

14 Coumel P, Cabrol C, Fabiato A, et al. Tachycardie permanente par rhythm réciproque. Arch Mal Coeur 1967, 60:1830-64.

15 Critelli G, Gallagher JJ, Monda V, et al. Anatomic and electro-physiologic substrate of the permanent form of junctional reciprocating tachycardia. 7 Am Coll Cardiol 1984;4:601-10.

16 Gillette PC, Garson A. Electrophysiologic and pharmacologic characteristics of autonomic ectopic atrial tachycardia. Circulation 1977;56:571-5.

17 Paladini D, Chita SK, Allan LD. Prenatal measurement of cardiothoracic ratio in evaluation of heart disease. Arch Dis Child 1990;65:20-3.

18 Allan LD, Anderson RH, Sullivan ID, et al. Evaluation of fetal arrhythmias by echocardiography. Br Heart $\mathcal{7} 1983 ; 50$. 240-5.

19 Kleinman CS, Donnerstein RL, Jaffe CC, et al. Fetal echocardiography: a tool for evaluation of in utero cardiac arrhythmias and monitoring of in utero therapy. Analysis of 71 patients. Am f Cardiol 1983;51:237-43.
20 Silverman NH, Enderlein MA, Stanger P, et al. Recognition of fetal arrhythmias by echocardiography. $\mathcal{F}$ Clin Ultrasound 1985;13:255-63.

21 Reed KL, Sahn DJ, Marx GR, et al. Cardiac Doppler flows during fetal arrhythmias: physiologic consequences. Obstet Gynecol 1987;70:1-6.

22 Strasburger JF, Huhta JC, Carpenter RJ, et al. Doppler echocardiography in the diagnosis and management of persistent fetal arrhythmias. F Am Coll Cardiol 1986;7:138691 .

23 DiMarco JP, Sellers TD, Lerman BB, et al. Diagnostic and therapeutic use of adenosine in patients with supraventricular tachyarrhythmias. F Am Coll Cardiol 1985;6:41725.

24 Clarke B, Till J, Rowland E, et al. Rapid and safe termination of supraventricular tachycardia in children with adenosine. Lancet 1987;i:299-301.

25 McIntosh-Yellin NL, Drew BJ, Scheinman MM. Safety and efficacy of central intravenous bolus administration of adenosine for termination of supraventricular tachycardia. 7 Am Coll Cardiol 1993;22:741-5.

26 Ralston MA, Knilans TK, Hannon DW, et al. Use of adenosine for the diagnosis and the treatment of tachyarrhythmias in pediatric patients. F Pediatr 1994;124:139-43.

27 Kohl T, Tercanli S, Kececioglu D, et al. Direct fetal administration of adenosine for the termination of sustained supraventricular tachycardia. Obstet Gynecol 1995;85:8734.

28 Houyel L, Fournier A, Ducharme G, et al. Electrophysiologic effects of sotalol on the immature mammalian heart. f Cardiovasc Pharmacol 1992;19:134-9.

29 Meden H, Neeb U. Transplazentare Kardioversion bei fetaler supraventrikulärer Tachykardie mit Sotalol. $Z$ Geburtsh Perinatol 1990;194:182-4.

30 Colin A, Chabaud JJ, Ponsoit J, et al. Les tachycardies supraventriculaires foetales et leur traitement. Arch Fr Pediatr 1989;46:335-40.

31 Maragnès $\mathrm{P}$, Tipple $\mathrm{M}$, Fournier $\mathrm{A}$. Effectiveness of oral sotalol for treatment of pediatric arrhythmias. Am $\mathcal{F}$ Cardiol 1992;69:751-4.

32 Pfammatter JP, Paul T, Lehmann C, et al. Efficacy and proarrhythmia of oral sotalol in pediatric patients. $7 \mathrm{Am}$ Coll Cardiol 1995;26:1002-7.

33 Allan LD, Chita KS, Sharland GK, et al. Flecainide in the treatment of fetal tachycardias. Br Heart f 1991;65:46-8.

34 Meijboom EJ, van Engelen AD, van de Beek EW, et al. Fetal arrhythmias. Curr Opin Cardiol 1994;9:97-102. 